\title{
Laparoscopic Surgery for Rectal Cancer: Approaches, Challenges and Outcome
}

\author{
Emad H. Aly \\ Aberdeen Royal Infirmary, Scotland \\ United Kingdom
}

\section{Introduction}

Jacobs et al (1991) reported the first series of laparoscopic colonic resections in 20 patients. It took over a decade of debate and controversy to establish that laparoscopic colon surgery for cancer, when compared with open surgery, is associated with better short term outcomes while maintaining at least equivalent long-term outcomes (Lee, 2009). These improved short term outcomes include reduced postoperative pain, short hospital stay, decreased intraoperative blood loss, quicker return of gut function and faster return to normal activities (Lacey et al., 2002; Veldkamp et al. ; 2005, Guillou et al., 2005). However, the debate around laparoscopic rectal surgery is far from over.

Oncologic outcomes of rectal cancer patients have been shown to depend on the skills and techniques of the operating surgeon (Heald et al., 1998). It took the surgical community several years to accept and adopt the current modern concepts of rectal cancer surgery which involves the standardisation of surgical resection with total mesorectal excision (Heald et al; 1986) and adequate circumferential resection margin (Quirke et al., 1988). It seems that the surgical community has yet to go through another phase of development in the surgical management of rectal cancer to address the concerns whether the technical challenges of laparoscopy may further add to the complexity of the surgical technique that may result in variability in outcomes (Lee, 2009).

Even though there are several studies on the outcomes of laparoscopic rectal surgery, there is limited level 1 evidence in surgical literature (Kang et al., 2010). Safety and benefits associated with laparoscopic colon cancer surgery have been demonstrated in many prospective randomized trials. However the same benefits have not yet been clearly confirmed for laparoscopic rectal cancer surgery (Lee, 2009). The debate around laparoscopic rectal surgery has become even more complex with the slow, but steady, increase of the practice of robotic rectal surgery. To be universally accepted, minimally invasive approaches to rectal cancer need to demonstrate at least equivalent onlcologic \& safety results to open surgery combined with evidence of improved short term outcomes. In addition, minimally invasive approach should be cost-effective (Champagne et al., 2007). 


\section{Conventional laparoscopic rectal surgery}

\subsection{Pros and cons of conventional laparoscopic rectal surgery}

Laparoscopic surgery provides unique, unobstructed views of the rectal dissection not only for the operating surgeon but also to the entire surgical team. Magnification of the surgical field allows more precise dissection. The pneumoperitoneum assists in opening up the planes for mobilization of the mesorectum (Lee, 2009). Also, blood loss is minimised as meticulous haemostasis is essential to preserve the laparoscopic view. (Cecil et al., 2006).

However, there are many well documented limitations to conventional laparoscopic rectal surgery. These include tremor, assistant dependant unstable two-dimensional view, inability to perform high-precision suturing, poor ergonomics and fixed tips as well as limited dexitry of surgical instruments (Delaney et al., 2003; Choi et al., 2009; Abodeely et al, 2010). These limitations are particularly relevant for rectal cancer surgery. As the surgeon operates into the confines of the pelvis the dissection becomes more difficult due to a combination of factors. Retraction of the rectum may prove difficult as one approaches the retrorectal and Denovillier's fascias. Crowding and clashing of instruments can result in a poor view and an experienced assistant is essential. Diathermy or vapour fumes from energy sources in the confined spaces of the pelvis often results in fogging of the camera scope that could slow progress of the procedure (Cecil et al., 2006).

\subsection{Challenges in conventional laparoscopic rectal surgery}

There are many technical challenges that are associated with laparoscopic rectal cancer surgery. One of the main technical challenges is obtaining adequate exposure, tissue tension \& retraction of the rectum by the currently available conventional laparoscopic instruments. In the absence of tactile feedback during laparoscopic surgery, intra-operative localisation of the tumour represents another challenge. Lesions during laparoscopic colon surgery are easily identifiable with or without tattoo marks. However, this is not easily possible with rectal cancers without tactile feedback. It is often difficult to determine the extent of distal rectal dissection that is needed to grantee adequate tumour clearance and to be sure that the stapler is applied at the appropriate level distal to the tumour (Lee, 2009).

There are well known limitations in the currently available laparoscopic distal rectal stapling devices. Most of the current laparoscopic staplers can only reticulate to a maximum of 65 degrees which makes horizontal division of the rectum difficult. For this reason, multiple firings are often required to complete distal rectal transection (Cecil et al., 2006). Division of the rectum in the presence of very low anterior and bulky lesions is particularly challenging (Champagne et al., 2011). Laparoscopic division of the distal rectum is not always technically feasible because of the limited angulation of the stapling device and the physical limitations of working in the bony confines of the pelvis. A virtual simulation demonstrated that the current design of staplers has to go through the iliac bone in order to achieve a $90^{\circ}$ angle at the level of levator ani (Brannigan et al., 2006). Some surgeons overcome by this by the use of a conventional stapler through a small suprapubic incision (Shalli et al., 2009).

Identifying the correct surgical plane anterior to Denonvillier's fascia during conventional laparoscopic rectal surgery, adequate radial margin and maintaining meticulous 
haemostasis are essential for high quality rectal cancer surgery. This can be challenging as without adequate retraction and tissue tension, surgical planes can be more ambiguous. Any bleeding will further obscure the appropriate anatomy (Champagne et al., 2011).

\subsection{Outcomes of conventional laparoscopic rectal surgery}

\subsubsection{Evidence from early randomised controlled trials}

The MRC CLASICC (Conventional vs. Laparoscopic- Assisted Surgery in Colorectal Cancer) Trial was done between 1996 and 2002 in 27 UK centres. It randomised 794 patients with colon and rectal cancer into laparoscopic resection $(n=526)$ open resection $(n=268)$ with a ratio of 2:1. The CLASICC trial was the first RCT to include patients with rectal cancer. The study reported a $29 \%$ conversion rate. Patients who had conversion ended up with increased complication rates. Also, there was higher incidence of positive circumferential resection margin after laparoscopic anterior resection but this did not reach statistical significance. There was no difference in hospital mortality or quality of life at 2 weeks and 3 months postoperatively (Guillou et al., 2005).

A subset of 148 patients in the CLASICC trial was analyzed with regard to sexual and urinary function with the help of validated questionnaires. The perceived change in the overall level of sexual function was considerably higher in the laparoscopic group compared with the conventional group (41 vs $23 \%$ ). These differences in sexual function reflected a clear trend, but were not significant at the $5 \%$ level.

Multivariate analysis revealed that conversion to open surgery was significantly correlated with postoperative male sexual dysfunction. In this study morbidity was an obvious issue in rectal surgery. Therefore, the authors concluded: 'laparoscopic resection for colon cancer is as effective as open surgery. However, impaired short-term outcomes after laparoscopic resection for rectal cancer do not yet justify its routine use. ' (Guillou et al., 2005).

\subsubsection{Challenges in rectal surgery as identified from the clasicc trial}

The outcomes of the MRC CLASICC trial should be interpreted with caution as in the study design, which was based on the best available data at that time (Simons et al., 1995), the surgeons' learning curve was set at 20 laparoscopic resections. Clearly this was an underestimation of the learning curve especially for rectal surgery (Leung et al., 2004; Good et al., 2011). This could explain the increased complication rate for laparoscopic rectal resections in the study and the authors' conclusion that laparoscopic rectal surgery was associated with increased morbidity. The reduction in the conversion rates for every year of the study is an indication that the learning curve was operative during the trial (Guillou et al, 2005). More recent studies set the learning curve at 55 cases for right-sided and 62 for leftsided laparoscopic colonic resections to achieve proficiency (Tekkis et al., 2005). However, there is little data on the numbers required to achieve proficiency in laparoscopic rectal cancer surgery (Good et al., 2011).

Despite the limitations of the MRC CLASICC trial, it helped to highlight the challenges of laparoscopic rectal surgery when compared to laparoscopic colon surgery. These findings were confirmed \& debated in other publications (Quah et al., 2002; Scheidbach et al., 2002; Cecil et al., 2006). It became evident that laparoscopic rectal surgery has a longer learning 
curve \& higher conversion rate, increased circumferential margin involvement as well as poorer outcome of urinary and sexual function especially in men (Quah et al., 2002; Bretagnol et al., 2005).

Increased anastomotic leak rate was another challenge that was identified in laparoscopic rectal surgery. Multiple firings are often required to complete distal rectal transection as the current laparoscopic staplers can only reticulate to a maximum of 65 degrees and therefore horizontal division of the rectum difficult. This is even more challenging for low-lying lesions and may contribute to increased anastomotic leakage. There is a documented positive relationship between the number of linear endostapler firings and anastomotic leak rate (Ito et al., 2008; Kim et al., 2009). Reported leak rate of $17 \%$ if the anastomosis were located less than $12 \mathrm{~cm}$ from the anal verge and as high as $25 \%$ in those patients who were not diverted following laparoscopic rectal surgery (Morino et al., 2003). These leak rates are higher than those reported after open rectal resection (4-11\%) (Enker et al., 1995; Heald et al. 1998). Refinements in the laparoscopic stapling devices may help addressing this problem in the future.

\subsubsection{Evidence from more recent publications}

More recent publications show much lower rate of circumferential resection margin (CRM) involvement following conventional laparoscopic rectal surgery between $2.14 \%-4 \%(\mathrm{Ng} \mathrm{K}$. H. et al., 2009; Lujan et al., 2009; Ng SS et al., 2009). On the other hand, anastomotic leak rate remains high for most of the studies in the range of 9 to $17 \%$ compared to $5 \%$ for open surgery (Champagne et al., 2007). However, some authors report anastomotic leak rate after conventional laparoscopic rectal surgery as low as 3. $8 \%$ (Good et al., 2011).

\subsubsection{Evidence from meta-analysis and cochrane reviews}

The CRM involvement was evaluated in a meta-analysis of 10 trials reported a mean positive radial margin of $5 \%$ for laparoscopic resections compared with $8 \%$ for open resections. However, this was not statistically significant. The distal margin positivity rates were also not different between laparoscopic and open rectal surgery (Anderson et al., 2008). However, it should be noted that a clear distal margin can be of an issue in laparoscopic resections of the lower third of the rectum, especially in male patients (Laurent et al., 2007).

A meta-analysis for 20 studies published between 1993 and 2004 looking at laparoscopic versus open surgery for rectal cancer showed that there was no significant difference in circumferential margin positivity or number of lymph nodes harvested. This confirmed that laparoscopic and open surgery were comparable in terms of their adequacy of oncological clearance (Aziz et al., 2006). Despite the known limitations of meta-analysis of nonrandomized data, the authors' conclusions are particularly useful because of the limited number of randomized data available for laparoscopic rectal cancer surgery (Champagne et al., 2007).

A Cochran systematic review for 48 studies comparing laparoscopic and open resection for rectal cancer concluded that laparoscopic TME surgery had clinically measurable short-term advantages in patients with primary resectable rectal cancer (Breukink et al., 2006). 


\subsubsection{Outcomes of conventional laparoscopic resection of extra-peritoneal rectal cancer in recent studies}

The ongoing debate as to the exact length of the rectum makes it difficult to accurately assess the results of the various studies reporting the outcomes of laparoscopic rectal surgery. Despite these apparent discrepancies there is an agreement that rectal cancer consists of extra-peritoneal and intra-peritoneal lesions and those tumours at or below the peritoneal reflection ideally should be grouped together (Champagne et al., 2011) when discussing laparoscopic rectal cancer outcomes because those lesions represent the real challenge for conventional laparoscopic rectal surgery. There is only limited number of studies that specifically address short and long term outcomes of conventional laparoscopic resection of these lesions.

\section{Short-term Outcomes:}

COREAN Trial (Comparison of Open versus laparoscopic surgery for mid and low REctal cancer After Neoadjuvant chemoradiotherapy)

This randomised study by Kang et al. (2010) aimed to evaluate the safety and short-term efficacy of laparoscopic surgery for rectal cancer after preoperative chemoradiotherapy. It was done between 2006-2009 in three tertiary referral centres by teams who have extensive experience in open and laparoscopic rectal surgery. Short-term outcomes assessed were involvement of the circumferential resection margin, macroscopic quality of the total mesorectal excision specimen, number of harvested lymph nodes, recovery of bowel function, perioperative morbidity, postoperative pain, and quality of life. Patients were followed up to assess the 3-year disease-free survival.

Conversion rate was 1. 2\%. Laparoscopic group had a longer operating time but less postoperative pain, decreased blood loss and better physical functioning score. There was no difference between the two groups in the involvement of the circumferential resection margin, macroscopic quality of the total mesorectal excision specimen, number of harvested lymph nodes, and peri-operative morbidity did not differ between the two groups. The authors concluded that 'Laparoscopic surgery after preoperative chemoradiotherapy for mid or low rectal cancer is safe and has short-term benefits compared with open surgery; the quality of oncological resection was equivalent' (Kang et al., 2010).

Even though the COREAN study showed no statistical difference in the anastomotic leak rate between the open and laparoscopic groups, other recent studies have highlighted that laparoscopic resection of extraperitoneal rectal cancer is associated with increased anastomotic leak rate when compared to open surgery. Reported anastomotic leak rate following conventional laparoscopic low anterior resection is 9\% (Morino et al., 2003; zhu et al., 2010) and up to $43 \%$ for an ultralow anterior resection (Choi et al., 2010).

\section{Long-term Outcomes:}

There is limited number of studies looking at the long-term prognosis following laparoscopic surgical resection of middle and lower rectal cancers. The long term outcomes in this subgroup of patients remain unclear because they are affected by anatomical factors as well as the complexity of the surgical procedures. Li et al. (2011) reported the outcome of 236 patients (laparoscopic, $n=113$; open, $n=123$ ) who underwent curative resection for middle and lower rectal cancer from 2000 to 2005. Surgery was performed by the same 
surgical team with extensive experience in laparoscopic and open procedures. The mean follow-up time of all patients was 74.8 months. There was no statistical differences in local recurrence, distant recurrence or the 5-year overall survival rates between the laparoscopic and open groups. The authors concluded that laparoscopic and open surgery for middle and lower rectal cancer offer similar long-term outcomes. The continued use of laparoscopic surgery in these patients can be supported'.

\section{Hybrid and hand-assisted laparoscopic rectal surgery}

In an attempt to avoid compromising oncologic rectal dissection but at the same time maintain the benefits of laparoscopic surgery some have studies reported the use of hybrid procedures in which colonic portion of the surgery is completed by the laparoscopic approach but rectal dissection is completed through a limited low midline or Pfannenstiel incision (Vithiananthan et al., 2001; Shalli et al., 2009).

Similarly, hand-assisted laparoscopic techniques have been used for rectal cancer surgery. Rectal exposure and dissection can be either performed directly through the hand assisted port incision using the open approach following laparoscopic mobilisation of the colon or laparoscopically with manual assistance. The latter technique uses the benefits of the unmatched laparoscopic view and at the same time allow the completion of oncologically similar dissection under tension as in open surgery (Lee et al., 2007).

Hybrid laparoscopic surgery allows the preservation of tactile sensation; optimal traction and exposure of the surgical planes and at the same time helps tumour localization which is a well known challenge in purely laparoscopic approach. Outcomes with this technique have been reported to be favourable and it certainly has some advantages but many surgeons feel that it is not 'totally' laparoscopic rectal surgery and it should not be included in trials or case series for laparoscopic rectal resection. However, the published literature highlights its role and it is suggested that if this procedure continues to demonstrate favourable outcomes and has a shorter learning curve it may require its own procedure code in the future (Champagne et al., 2011).

\section{Robotic assisted rectal surgery}

The inherent limitations of conventional laparoscopic surgery; which include tremor, unstable two-dimensional view, and limited degree of freedom of the instruments, are particularly significant in patients undergoing rectal cancer surgery with total mesorectal excision (Ballantyne, 2002). Robotic surgery has the potential to address some of these limitations and the potential to offer technical abilities greater than those offered by open or conventional laparoscopic surgery. Robotic colorectal surgery was first reported in by Ballantyne et al. in 2001. The concept of robotic total mesorectal excision for rectal cancer was first reported by Pigazzi et al. in 2006.

\subsection{Potential advantages of robotic surgery}

Robotic systems can address many of the inherent limitations of conventional laparoscopic surgery. These include the fulcrum effect, poor depth perception, limited range of motion as wells as instrument tremor (Maeso et al., 2010). The use of robotic systems in rectal cancer 
surgery has the potential to offer accurate dissection in the avascular 'holly' plane of the rectum without injury to the integrity of the mesorectum or autonomic pelvic nerves in the narrow pelvic cavity. Ultralow rectal dissection could be more easily performed by using precise movements of robotic arms (Kim \& Kang, 2010).

Robotic system offers a camera system that is controlled by the operating surgeon combined with three dimensional ten-fold magnification vision thus allowing a perfectly still visibility of the operative field. (Ballantyne \& Moll, 2003; Lanfranco et al., 2004; Baik, 2008). One of the main advantages of the use of robotic system in rectal surgery is that it gives the surgeon equal access to both right and left pelvis as if standing above the head of the patient (Hellan et al., 2007). The tips of the instruments of a robotic arm have an endowrist which has functions of seven degrees of freedom, one hundred and eighty degrees articulation and five hundred and forty degrees rotation (Baik, 2008). Also the availability of multi-articulated instruments allows for a range of angles to approach the rectum thus allowing sharp dissection around the rectum and mesorectum. The robotic handles transfer the hand movements of the surgeon to the tip of the instruments offering a comfortable, ergonomically ideal operating position (Stylopoulos \& Rattner, 2003). Many studies highlighted that the operating surgeon experienced less physical strain with robotic assisted surgery (Stylopoulos \& Rattner, 2003; Ballantyne et al., 2003; Hellan et al., 2007). In addition, robotic technology offers additional features such as motion scaling and remote telesurgical applications including tele-mentoring (Kim \& Kang, 2010)

\subsection{Limitations of the current robotic systems}

The current robotic systems have some limitations which should be addressed in the future. There is lack of both tactile sensation and tensile feedback to the operating surgeon. Therefore, tissue damage can occur easily during traction by the robotic arm and during movement of the robotic instrument (Baik, 2008). Therefore, the surgeon must rely on visual cues to estimate the amount of tension exerted on the tissues (Hellan et al., 2007). Also, suture material can be cut during suturing because there is no tensile feedback to the robotic instrument (Baik, 2008). Therefore, great care must be taken to avoid traumatic injuries when handling bowel.

The system requires precise positioning of the robot for optimal operative outcome and to avoid robotic arm collision and the position of the patient cannot be changed without undocking the robotic arms (Hellan et al., 2007). The docking and separation procedure of a robotic cart from the patient is a time consuming procedure. Also, when using the robot to perform a surgical procedure in different compartments in the abdominal cavity, such as anterior resection, repeated docking and undocking of the robot is often needed and this is reflected on increased operating time. When immediate open conversion is necessary to deal with serious intra-operative bleeding delayed separation of the robotic cart can create a difficult situation (Baik, 2008).

High capital and running costs of the currently available robotic system made the taking up of this technology rather limited in many countries. The average price of one robotic system is more than $\mathrm{U} \$ 2,000,000$ and combining this with $\mathrm{U} \$ 2,000$ for the disposable instruments is a major issue when cost-effectiveness of robotic surgery is debated (Baik, 2008). 


\subsection{Approaches to robotic rectal surgery}

There are several techniques for robotic rectal cancer surgery described in the surgical literature.

\subsubsection{Multiple stages totally robotic technique}

This is described as either a two-stage technique or a three-stage technique (D'Annibale et al., 2004). The number of stages mirrors the number of movements of the robotic cart. The need for frequent docking \& undocking of the robotic system is reflected on increased operating time.

\subsubsection{Hybrid technique}

To eliminate the need for repositioning of the robot most surgeons choose conventional laparoscopic mobilization of the left colon and splenic flexure as well as division of the inferior mesenteric vessels and then use the robot for the TME part of the operation only. This hybrid approach saves the time for repetitive robotic setups. The actual TME is reported to take an average of 60 minutes (Hellan et al., 2007; Baik et al., 2008).

\subsubsection{Single stage totally robotic}

This approach has been recently reported by Kawak \& Kim (2011). It eliminates the need for frequent docking \& undocking of the robot but at the same time preserves the advantages of the use of the robotic approach for the whole procedure.

\subsection{Advantages of totally robotic rectal surgery}

Advocates of the totally robotic approach, whether multiple stage or single stage, believe that robotic dissection around the IMA pedicle is a fundamental step of the procedure to identify and preserve the periaortic nerves. They correctly consider that not only the pelvic nerves but also the periaortic nerves are important in sexual/bladder functions. They also feel that the use of robotics could also improve access and enable easier mobilization of the splenic flexure (Kawak \& Kim, 2011).

\subsection{Learning curve in robotic rectal surgery}

The three-dimensional view and the ability of the robot to transfer the surgeon's hand movements to the tips of the surgical instruments makes the learning curve for robotic surgery much less steep than that for laparoscopic colorectal surgery. Therefore, an inexperienced laparoscopic surgeon is able to operate with the robot safely (Hellan et al, 2007). This is particularly evident for distal rectal cancers as the robot offers superior visualization and mobility during the pelvic dissection (Abodeely et al., 2010). Robotic surgery actually requires the same skill set used during open surgery and thus the learning curve at the console is relatively short (Giulianotti et al., 2003).

The learning curve of robotic assisted technology entails the surgeon's mastery of several unique skills to overcome the loss of tensile and tactile feedback by recognizing visual cues, conceptualize the spatial relationships of robotic instruments outside the active field of view 
and mentally visualizing the spatial relationships of the robotic arms and cart while operating at the console (Bokhari et al., 2011).

Bokhari et al. (2011) believe that to facilitate the acquisition of robotic unique skills in a safe and stepwise manner, the surgeon should acquire expert laparoscopic skills before using the robotic approach. They divided the learning curve into three phases. Phase 1 to include 15 cases and it represents the initial part of the learning curve. Phase 2 includes an additional 10 cases to allow the consolidation of the additional experience once the initial learning curve has been completed. Phase 3 is the post-learning period when the surgeon can start offering robotic surgery for a more complex and challenging cases. The authors concluded that their data suggest that after a learning curve phase that involves of 15 to 25 cases, the surgeon may achieve a higher level of competence and consider using robotic surgery for patients presenting with more difficult cases safely.

\subsection{Outcomes of robotic assisted rectal surgery}

\subsubsection{Evidence from case series reports \& case-matched studies}

Pigazzi et al. (2006) reported the first series of robotic-assisted low anterior resection with total mesorectal excision on six consecutive patients with rectal cancer. These cases were compared with six consecutive low anterior resections performed with conventional laparoscopic technique by the same surgeon. There were no conversions in either group. Operative and pathological data, complications, and hospital stay were similar in both groups. However, robotic operations appeared to cause less strain for the operating surgeon. The authors concluded that robotic-assisted laparoscopic low anterior resection for rectal cancer is feasible in experienced hands and the technique may facilitate minimally invasive radical rectal surgery. The same group published 2 further case-matched reports with bigger series (Hellan et al., 2007; Baek et al., 2011) confirming their conclusion from the initial study. They particularly highlighted the low conversion rate with robotic assisted rectal surgery.

Patriti et al. (2009) reported on the short and medium term outcome of robotic assisted and traditional laparoscopic rectal resection in a case matched study. They found that robotassisted laparoscopic rectal resection results in shorter operative time when a total mesorectal excision is performed and significantly lowers conversion rate. Postoperative morbidity was comparable between both groups. Overall survival and disease-free survival were comparable between groups, even though a trend towards better disease-free survival in the robotic assisted group was observed.

Baik et al. (2009) compared the short-term results between robotic-assisted low anterior resection and standard laparoscopic low anterior resection in rectal cancer. Conversion rate and serious complication rate were significantly lower in the robotic surgery group. The specimen quality was acceptable in both groups with a significantly better quality of the mesorectum grading in the robotic group.

Biffi et al. (2011) investigated the estimated blood loss after full robotic low anterior resection in a case-matched model with conventional open approach. Estimated intraoperative blood loss was significantly higher in the open group. They also found that the robotic surgery group had significantly decreased length of hospital stay, increased number of harvested lymph nodes and extent of distal margin. 
Pigazzi et al. (2010) reported a multicentre study on the role of robot-assisted tumourspecific rectal surgery (RTSRS) to verify, on a multicentre basis, the peri-operative and oncologic outcome of RTSRS. The study included 140 consecutive patients undergoing RTSR in three centres. Conversion rate was $4.9 \%$. The number of harvested nodes and margin status compared favourably with those of open series. The 3-year overall survival rate was $97 \%$ with no isolated local recurrences were found at mean follow-up of 17.4 months. The authors concluded that RTSRS is a safe and feasible procedure and highlighted the need for randomized clinical trials and longer follow-up are needed to evaluate a possible influence of RTSRS on patient survival.

Park et al. (2011) compared the short-term outcomes in 263 patients (open group $=88$ patients, laparoscopic group $n=123 \&$ robot-assisted group $n=52$ ). Patients from the laparoscopic \& robotic assisted groups recovered significantly faster than did those from the open surgery group. The specimen quality, with a distal resection margin, harvested lymph nodes, and circumferential margin, did not differ among the three groups. The authors concluded that laparoscopic \& robotic assisted surgery reproduce equivalent short-term results of standard open surgery while providing the advantages of minimal access. For experienced laparoscopic colorectal surgeon, the use of the da Vinci robot resulted in no significant short-term clinical benefit over the conventional laparoscopic approach.

\subsubsection{Evidence from randomised controlled trials (RCT)}

There is lack of evidence from RCT to support robotic assisted surgery for rectal cancer. The RObotic versus LAparoscopic Resection for Rectal cancer (ROLARR) is designed to address this issue. This is an international, multicentre, prospective, randomised, controlled, unblinded, parallel-group trial of robotic-assisted versus laparoscopic surgery for the curative treatment of rectal cancer.

The study will perform a rigorous evaluation of robotic-assisted rectal cancer surgery against conventional laparoscopic rectal cancer resection by means of a randomised, controlled trial. Key short-term outcomes will include assessment of technical ease of the operation and improved oncological outcome. Also, quality of life assessment and analysis of cost-effectiveness will be performed. Short-term outcomes will be analysed to provide a timely assessment of the new technology. Longer-term outcomes will focus on oncological aspects of the disease with analysis of disease-free and overall survival and local recurrence rates at 3-year follow-up (http://www. leeds. ac. uk/hsphr/research/AUHE/rolarr. html).

\section{Extended resection techniques in laparoscopic rectal cancer surgery}

In an attempt to decrease the risk of local recurrence following radical surgery for rectal cancer, techniques have been described which offer extended resection. These were originally described in open surgery, but more recently these extended resections have been also offered laparoscopically (Georgiou et al., 2009; Stelzner et al., 2011).

\subsection{Laparoscopic extended lateral pelvic node dissection}

Lateral pelvic lymph node involvement in rectal cancer has been well recognised for many years (Sauer et al., 1951). In Japan, lateral pelvic node dissection is performed to minimise 
local recurrence and improve survival as they believe that total mesorectal excision does not clear the metastases in the lateral pelvic lymph nodes which has an overall incidence between 8. 6 to 27\% (Fujita et al., 2003; Kim et al., 2008). However, evidence from metaanalysis confirms that extended lateral pelvic node dissection is associated with increased blood loss and urinary and sexual dysfunction without significant overall cancer-specific advantage (Georgiou et al., 2009). Therefore, preoperative radiotherapy or chemoradiotherapy followed by rectal resection with total mesorectal excision is the standard treatment in Western countries.

There is limited evidence in the literature on the outcomes of laparoscopic extended lateral pelvic node dissection with total meseorectal excision in patients with locally advanced rectal cancer. Advocates of the technique believe that laparoscopic approach has the potential to decrease the well known drawbacks of the open lateral pelvic wall dissection as the magnified views offer clear vision of the smallest structures in the narrow pelvis which make it easier to identify lymphatic tissue and protect the autonomic nerve plexus (Park et al., 2011). Some studies also suggest decreased blood loss and operative time with the laparoscopic approach (Fujita et al., 2003). Increasingly, robotic assisted approach has been used to perform extended lateral pelvic wall lymphadenectomy with remarks that dissection of the lymph nodes around major pelvic vessels is much easier (Park et al., 2011).

However, the currently available evidence is rather limited to make valid conclusions on the safety and outcomes of laparoscopic or robotic-assisted extended lateral pelvic node dissection in locally advanced rectal cancer.

\subsection{Laparoscopic extended abdominoperineal resection for low rectal cancer}

Standard abdominoperineal resection (APR) is well known to be associated with inferior oncological outcomes when compared to anterior resection for low rectal cancer (Stelzner et al., 2011). This is attributed to the relatively high incidence of intra-operative tumour or bowel perforation and positive circumferential resection margin due to the relative lack of the mesorectal fat around the lower rectum (Nagtegaal et al, 2005). This led to progressive development of an extended version of APR, now known as extended abdominoperineal resection (EAP), extralevator abdominoperineal excision (ELAP) or cylindrical abdominoperineal resection (Holm et al, 2007; Marecik et al, 2011). This technique aims at a wider circumferential resection margin at the level of the tumour bearing segment. Systematic reviews suggests that extended techniques of APR result in superior oncologic outcome in terms of lower incidence of tumour perforation and positive circumferential resection margin when compared to standard techniques (Stelzner et al., 2011).

However, extended abdominoperineal resection has been reported to be associated with higher incidence of perineal wound complications and hernia in comparison with the conventional APR. Also, lateral relatively blind excision of the levator muscles can put the neurovascular structures along the lateral pelvic wall at risk for injury which could increase the morbidity of the procedure. There are limited reports in the literature on laparoscopic and robotic assisted extended abdominoperineal resection (Marecik et al, 2011). However, the data so far are limited and more research is needed to assess the specific value of laparoscopic and robotic-assisted approach in relation to extended abdominoperineal resection. 


\section{Other approaches for laparoscopic rectal cancer surgery}

There are few reports in the literature describing alternative approaches to rectal cancer surgey including single port access mesorectal excision (Gaujoux et al., 2011; Bulut et al., 2011; Lauritsen \& Bulut, 2011), transanal or transvaginal retrieval of the specimen (Choi et al., 2009). However, the number of studies \& cases are limited to make any valid conclusions. There is slow, but steadily increasing, interest in the application of completely natural orifice transluminal surgery (NOTES) in colorectal surgery (Sylla, 2010).

\section{Conclusion}

The debate over the optimal approach to the laparoscopic resection of rectal cancer is far from over. The short-term outcomes of conventional laparoscopic resections by experienced laparoscopic surgeons are better than those of open surgery and seem to be improving over time. The role of robotic assisted rectal surgery still needs to be better defined. There is limited evidence whether laparoscopic approach confers any long-term benefits for the patients. Also, further research is needed to establish if the different laparoscopic approaches can vary in their effect on the long term prognosis.

\section{References}

Abodeely, A.; Lagares-Garcia, J. A.; Duron, V.; Vrees M. (2010). Safety and learning curve in robotic colorectal surgery. J Robotic Surg; 4:161-165

Anderson, C.; Uman, G.; Pigazzi, A. (2008). Oncologic outcomes of laparoscopic surgery for rectal cancer: a systematic review and meta-analysis of the literature. Eur J Surg Oncol; 34(10)1135-42

Aziz, O.; Constantinides, V.; Tekkis, P.; et al. (2006). Laparoscopic versus open-surgery for rectal cancer: a meta-analysis. Ann Surg Onc;13:413-424

Baek, J. H.; Pastor, C.; Pigazzi, A. (2011). Robotic and laparoscopic total mesorectal excision for rectal cancer: a case-matched study. Surg Endosc; 25:521-525

Baik, S. H. (2008). Robotic colorectal surgery. Yonsei Med J.; 31; 49(6):891-6

Baik, S. H.; Lee, W. J.; Rha, K. H.; Kim, N. K.; Sohn, S. K.; Chi, H. S.; Cho, C. H.; Lee, S. K.; Cheon, J. H.; Ahn, J. B.; Kim, W. H. (2008). Robotic total mesorectal excision for rectal cancer using four robotic arms. Surg Endosc 22:792-797

Baik, S. H.; Kwon, H. Y.; Kim, J. S.; Hur, H; Sohn, S. K.; Cho, C. H.; Kim, H . (2009). Robotic versus laparoscopic low anterior resection of rectal cancer: short-term outcome of a prospective comparative study. Ann Surg Oncol.; 16(6):1480-7

Ballantyne, G. H.; Merola, P.; Weber, A.; Wasielewski, A. (2001). Robotic solutions to the pitfalls of laparoscopic colectomy. Osp Ital Chir;7:405-12

Ballantyne, G. H. (2002). Robotic surgery, telerobotic surgery, telepresence, and telementoring: review of early clinical results. Surg Endosc 16:1389-1402

Ballantyne, G. H.; Moll, F. (2003). The da Vinci telerobotic surgical system: the virtual operative field and telepresence surgery. Surg Clin North Am.; 83:1293-1304

Biffi, R.; Luca, F.; Pozzi, S.; Cenciarelli, S.; Valvo, M.; Sonzogni, A.; Radice, D.; Ghezzi, T. L. (2011). Operative blood loss and use of blood products after full robotic and conventional low anterior resection with total mesorectal excision for treatment of rectal cancer. J Robot Surg.; 5(2):101-107 
Bokhari, M. B.; Patel, C. B.; Ramos-Valadez, D. I.; Ragupathi, M.; Haas, E. M.; (2011). Learning curve for robotic-assisted laparoscopic colorectal surgery. Surg Endosc.; 25(3):855-60

Brannigan, A. E.; De Buck, S.; Suetens, P.; Penninckx, F.; D’Hoore, A. (2006). Intracorporeal rectal stapling following laparoscopic total mesorectal excision: overcoming a challenge. Surg Endosc; 20: 952-955

Bretagnol, F.; Lelong, B.; Laurent, C.; et al. (2005). The oncological safety of laparoscopic total mesorectal excision with sphincter preservation for rectal carcinoma. Surg Endosc; 19 (7): 892-896

Breukink, S.; Pierie, J.; Wiggers, T. (2006). Laparoscopic versus open total mesorectal excision for rectal cancer. Cochrane Library, Cochrane Database of Systematic Reviews; $4: 1-79$

Bulut, O.; Nielsen, C. B.; Jespersen, N. (2011). Single-port access laparoscopic surgery for rectal cancer: initial experience with 10 cases. Dis Colon Rectum.; 54(7):803-9

Cecil, T. D.; Taffinder, N.; Gudgeon, A. M. (2006). A personal view on laparoscopic rectal cancer surgery. Colorectal Dis; 8: 30-2

Champagne, B. J.; Delaney, C. P. (2007). Laparoscopic approaches to rectal cancer. Clin Colon Rectal Surg; 20(3):237-48

Champagne, B. J.; Makhija, R. (2011). Minimally invasive surgery for rectal cancer: are we there yet? World J Gastroenterol; 17(7):862-6

Choi, D. H.; Hwang, J. K.; Ko, Y. T.; Jang, H. J.; Shin, H. K.; Lee, Y. C.; Lim, C. H.; Jeong, S. K.; Yang, H. K. (2010). Risk factors for anastomotic leakage after laparoscopic rectal resection. J Korean Soc Coloproctol.; 26(4):265-73

Choi, G. S.; Park, I. J.; Kang, B. M.; Lim, K. H.; Jun, S. H. (2009). A novel approach of roboticassisted anterior resection with transanal or transvaginal retrieval of the specimen for colorectal cancer. Surg Endosc (2009) 23:2831-2835

D'Annibale, A.; Morpurgo, E.; Fiscon, V. et al. (2004) Robotic and laparoscopic surgery for treatment of colorectal diseases. Dis Colon Rectum 47:2162-2168

Delaney, C. P.; Lynch, A. G.; Senagore, A. J.; Fazio, V. W. (2003). Comparison of robotically performed and traditional laparoscopic colorectal surgery. Dis Colon Rectum; 46:1633-9

Enker, W. E.; Thaler, H. T.; Cranor, M. L.; Polyak, T. (1995). Total mesorectal excision in the operative treatment of carcinoma of the rectum. J Am Coll Surg; 181(4):335-346

Gaujoux, S.; Bretagnol, F.; Au, J.; Ferron, M.; Panis, Y. (2011). Single port access proctectomy with total mesorectal excision and intersphincteric resection with a primary transanal approach. Colorectal Dis.; 13(9):e305-7

Georgiou, P.; Tan, E.; Gouvas, N.; Antoniou, A.; Brown, G.; Nicholls, R. J.; Tekkis, P. (2009). Extended lymphadenectomy versus conventional surgery for rectal cancer: a metaanalysis. Lancet Oncol. 2009; 10:1053-62

Giulianotti, P. C.; Coratti, A.; Angelini, M.; et al. (2003). Robotics in general surgery: personal experience in a large community hospital. Arch Surg; 138:777-84

Good, D. W.; O'Riordan, J. M.; Moran, D.; Keane, F. B.; Eguare, E.; O'Riordain, D. S.; Neary, P. C. 2011. Laparoscopic surgery for rectal cancer: a single-centre experience of 120 cases. Int J Colorectal Dis. 2011 Jun 24. [Epub ahead of print]

Guillou, P. J.; Quirke, P.; Thorpe, H.; Walker, J.; Jayne, D. G.; Smith, A. M. et al. ( 2005). MRC CLASICC Trial Group. Short-term endpoints of conventional versus 
laparoscopicassisted surgery in patients with colorectal cancer (MRC CLASICC trial): multicentre, randomised controlled trial. Lancet; 365: 1718-26

Fujita, S.; Yamamoto, S.; Akasu, T.; Moriya, Y. (2003). Lateral pelvic lymph node dissection for advanced lower rectal cancer. Br J Surg; 90:1580-1585. Heald, R. J.; Ryall, R. D. (1986). Recurrence and survival after total mesorectal excision for rectal cancer. Lancet; $28 ; 1$ (8496):1479-82

Heald, R. J.; Moran, B. J.; Ryall, R. D.; Sexton, R.; MacFarlane, J. K. (1998). Rectal cancer: the Basingstoke experience of total mesorectal excision, 1978-1997. Arch Surg; 133(8):894-899

Hellan, M.; Anderson, C.; Ellenhorn, J. D.; Paz, B.; Pigazzi, A. (2007). Short-term outcomes after robotic-assisted total mesorectal excision for rectal cancer. Ann Surg Oncol.; 14(11):3168-73

Holm, T.; Ljung, A.; Häggmark, T.; Jurell, G.; Lagergren, J. (2007). Extended abdominoperineal resection with gluteus maximus flap reconstruction of the pelvic floor for rectal cancer. Br J Surg; 94:232-238

Ito, M.; Sugito, M.; Kobayashi, A.; Nishizawa, Y.; Tsunoda, Y.; Saito, N. (2008),. Relationship between multiple numbers of stapler firings during rectal division and anastomotic leakage after laparoscopic rectal resection. Int J Colorectal Dis; 23:703-7

Jacobs, M.; Verdeja, J. C.; Goldstein, H. S. (1991). Minimally invasive colon resection (laparoscopic colectomy). Surg Laparosc Endosc 1: 144-50

Kang, S. B.; Park, J. W.; Jeong, S. Y.; Nam, B. H.; Choi, H. S. et al (2010). Open versus laparoscopic surgery for mid or low rectal cancer after neoadjuvant chemoradiotherapy (COREAN trial): short-term outcomes of an open-label randomised controlled trial. Lancet Oncol.; 11(7):637-45

Kim, T. H.; Jeong, S. Y.; Choi, D. H.; Kim, D. Y.; Jung, K. H.; Moon, S. H.; Chang, H. J.; Lim, S. B.; Choi, H. S.; Park, J. G. (2008). Lateral lymph node metastasis is a major cause of locoregional recurrence in rectal cancer treated with preoperative chemoradiotherapy and curative resection. Ann Surg Oncol; 15:729-737

Kim, J. S.; Cho, S. Y.; Min, B. S.; Kim, N. K. (2009). Risk factors for anastomotic leakage after laparoscopic intracorporeal colorectal anastomosis with a double stapling technique. J Am Coll Surg; 209:694-701

Kim, N. K. \& Kang, J. (2010). Optimal Total Mesorectal Excision for Rectal Cancer: the Role of Robotic Surgery from an Expert's View. J Korean Soc Coloproctol.; 26(6):377-87

Kwak, J. M., Kim, S. G. (2011). The technique of single-stage totally robotic low anterior resection. J Robotic Surg; 5:25-28

Lacy, A. M.; Garcia-Valdecasas, J. C.; Delgado, S.; Castells, A.; Taurá, P.; Piqué, J. M. et al. (2002). Laparoscopy-assisted colectomy versus open colectomy for treatment of nonmetastatic colon cancer: a randomized trial. Lancet; 359: 2224-9

Lanfranco, A. R.; Castellanos, A. E.; Desai, J. P.; Meyers, W. C. (2004). Robotic surgery: a current perspective. Ann Surg.; 239:14-21

Laurent, C.; Leblanc, F.; Gineste, C.; Saric, J.; Rullier, E. (2007). Laparoscopic approach in surgical treatment of rectal cancer. Br J Surg; 94(12):1555-61

Lauritsen, M. L.; Bulut, O. (2011). Single-port access laparoscopic abdominoperineal resection through the colostomy site: a case report. Tech Coloproctol. [Epub ahead of print] 
Lee, S. W.; Sonoda, T.; Milsom, J. W. (2007). Expediting of laparoscopic rectal dissection using a hand-access device. Dis Colon Rectum ; 50(6):927-929

Lee, S. W. (2009). Laparoscopic Procedures for Colon and Rectal Cancer Surgery. Clin Colon Rectal Surg; 22:218-224

Leung, K. L.; Kwok, S. P. Y.; Lam, S. C. W.; et al. (2004). Laparoscopic resection of rectosigmoid carcinoma: prospective randomised trial. Lancet; 363: 1187-92

Li, S.; Chi, P.; Lin, H.; Lu, X.; Huang, Y. (2011). Long-term outcomes of laparoscopic surgery versus open resection for middle and lower rectal cancer: an NTCLES study. Surg Endosc. 2011 Apr 13. [Epub ahead of print]

Lujan, J.; Valero, G.; Hernandez, Q.; Sanchez, A.; Frutos, M. D.; Parrilla P. (2009). Randomized clinical trial comparing laparoscopic and open surgery in patients with rectal cancer. Br J Surg; 96: 982-989

Maeso S, Reza M, Mayol JA, Blasco JA, Guerra M, Andradas E, et al. (2010) Efficacy of the Da Vinci surgical system in abdominal surgery compared with that of laparoscopy: a systematic review and meta-analysis. Ann Surg.;252:254-62

Marecik, S. J.; Zawadzki, M.; Desouza, A. L.; Park, J. J.; Abcarian, H.; Prasad, L. M. (2011). Robotic cylindrical abdominoperineal resection with transabdominal levator transection. Dis Colon Rectum; 54:1320-5

Morino, M.; Parini, U.; Giraudo, G.; Salval, M.; Brachet Contul, R.; Garrone C. (2003). Laparoscopic total mesorectal excision: a consecutive series of 100 patients. Ann Surg; 237(3): 335-342

Nagtegaal, I. D.; van de Velde, C. J.; Marijnen, G. C.; van Krieken, J. H. J. M., Quirke, P. (2005). Low rectal cancer: a call for a change of approach in abdominoperineal resection. J Clin Oncol 23:9257-9264

Ng, K. H.; Ng, D. C.; Cheung, H. Y.; Wong, J. C.; Yau, K. K.; Chung, C. C.; Li, M. K. (2009). Laparoscopic resection for rectal cancers: lessons learned from 579 cases. Ann Surg; 249: $82-86$

Ng, S. S.; Leung, K. L.; Lee, J. F.; Yiu, R. Y.; Li J. C.; Hon, S. S. (2009). Long-term morbidity and oncologic outcomes of laparoscopic-assisted anterior resection for upper rectal cancer: ten-year results of a prospective, randomized trial. Dis Colon Rectum; 52: 558-566

Park, J. S.; Choi, G. S.; Lim, K. H.; Jang, Y. S.; Jun, S. H. (2011). S052: a comparison of robotassisted, laparoscopic, and open surgery in the treatment of rectal cancer. Surg Endosc.; 25(1):240-8

Patriti, A.; Ceccarelli, G.; Bartoli, A.; Spaziani, A.; Biancafarina, A.; Casciola, L. (2009). Shortand medium-term outcome of robot-assisted and traditional laparoscopic rectal resection. JSLS.; 13(2):176-83

Pigazzi, A.; Ellenhorn, J. D.; Ballantyne, G. H.; Paz, I. B. (2006). Roboticassisted laparoscopic low anterior resection with total mesorectal excision for rectal cancer. Surg Endosc; 20:1521-5

Pigazzi, A.; Luca, F.; Patriti, A.; Valvo, M.; Ceccarelli, G.; Casciola, L.; Biffi, R.; GarciaAguilar, J.; Baek, J. H. (2010). Multicentric study on robotic tumor-specific mesorectal excision for the treatment of rectal cancer. Ann Surg Oncol.; 17(6):161420 
Quah, H. M.; Jayne, D. G.; Eu, K. W.; Seow-Choen, F. (2002). Bladder and sexual dysfunction following laparoscopically assisted and conventional open mesorectal resection for cancer. Br J Surg; 89:1551-1556

Quirke, P.; Dixon, M. F. (1988). The prediction of local recurrence in rectal adenocarcinoma by histopathological examination. Int J Colorectal Dis.; 3(2):127-31

Sauer, I.; Bacon, H. E. (1951). Influence of lateral spread of cancer of the rectum on radicability of operation and prognosis. Am J Surg; 81:111-120

Scheidbach, H.; Schneider, C.; Konradt, J. et al. (2002). Laparoscopic abdominoperineal resection and anterior resection with curative intent for carcinoma of the rectum. Surg Endosc; 16:7-13

Shalli, K.; MacDonald, E.; Aly, E. H. (2009). Towards lower complication rate in laparoscopic rectal surgery. Colorectal Disease, 11 (Suppl. 2), 55. Abstract

Simons, A. J.; Anthone, G. J.; Ortega, A. E.; et al. (1995). Laparoscopic-assisted colectomy learning curve. Dis Colon Rectum; 38: 600-03

Stelzner, S.; Koehler, C.; Stelzer, J.; Sims, A.; Witzigmann, H. (2011). Extended abdominoperineal excision vs. standard abdominoperineal excision in rectal cancer-a systematic overview. Int J Colorectal Dis.; 26(10):1227-40

Stylopoulos, N.; Rattner, D. (2003). Robotics and ergonomics. Surg Clin North Am.; 83:132137

Sylla; P. (2010). Current experience and future directions of completely NOTES colorectal resection. World J Gastrointest Surg.; 2(6): 193-198

Tekkis; P. P.; Senagore; A. J.; Delaney, C. P., Fazio, V. W. (2005). Evaluation of the learning curve in laparoscopic colorectal surgery: comparison of right-sided and left-sided resections. Ann Surg; 242(1):83-91

Veldkamp, R.; Kuhry, E.; Hop, W. C.; Jeekel, J.; Kazemier, G.; Bonjer, H. J. et al. (2005). Colon cancer Laparoscopic or Open Resection Study Group (COLOR). Laparoscopic surgery versus open surgery for colon cancer: short-term outcomes of a randomised trial. Lancet Oncol; 6: 477-84

Vithiananthan, S.; Cooper, Z.; Betten, K.; et al. (2001). Hybrid laparoscopic flexure takedown and open procedure for rectal resection is associated with significantly shorter length of stay than equivalent open resection. Dis Colon Rectum; 44(7):927-935

Zhu, Q. L.; Feng, B.; Lu, A. G.; Wang, M. L.; Hu, W. G.; Li, J. W.; Mao, Z. H.; Zheng, M. H. (2010). Laparoscopic low anterior resection for rectal carcinoma: complications and management in 132 consecutive patients. World J Gastroenterol.; 16(36):4605-10 


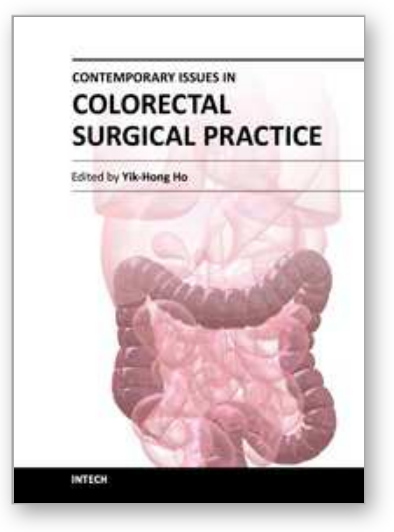

\author{
Contemporary Issues in Colorectal Surgical Practice \\ Edited by Dr. Yik- Hong Ho
}

ISBN 978-953-51-0257-1

Hard cover, 126 pages

Publisher InTech

Published online 16, March, 2012

Published in print edition March, 2012

In recent years, significant progress in colorectal surgery has been made which includes laparoscopic techniques, pre-operative management, emergency colorectal surgery, fast track multimodal recovery, management of complex wound problems and colorectal cancer follow-up. "Contemporary Issues in Colorectal Surgical Practice" aims to bridge the gap between the journal article and the traditional textbook in these areas.

\title{
How to reference
}

In order to correctly reference this scholarly work, feel free to copy and paste the following:

Emad H. Aly (2012). Laparoscopic Surgery for Rectal Cancer: Approaches, Challenges and Outcome, Contemporary Issues in Colorectal Surgical Practice, Dr. Yik- Hong Ho (Ed.), ISBN: 978-953-51-0257-1, InTech, Available from: http://www.intechopen.com/books/contemporary-issues-in-colorectal-surgicalpractice/laparoscopic-surgery-for-rectal-cancer-approaches-challenges-outcome

\section{INTECH}

open science | open minds

\section{InTech Europe}

University Campus STeP Ri

Slavka Krautzeka 83/A

51000 Rijeka, Croatia

Phone: +385 (51) 770447

Fax: +385 (51) 686166

www.intechopen.com

\section{InTech China}

Unit 405, Office Block, Hotel Equatorial Shanghai

No.65, Yan An Road (West), Shanghai, 200040, China 中国上海市延安西路65号上海国际贵都大饭店办公楼 405 单元

Phone: +86-21-62489820

Fax: $+86-21-62489821$ 
(C) 2012 The Author(s). Licensee IntechOpen. This is an open access article distributed under the terms of the Creative Commons Attribution 3.0 License, which permits unrestricted use, distribution, and reproduction in any medium, provided the original work is properly cited. 